\title{
A longitudinal study of serological responses to Coxiella burnetii and shedding at kidding among intensively-managed goats supports early use of vaccines
}

\author{
Michael Muleme ${ }^{1^{*}} \mathbb{D}$, Angus Campbell², John Stenos ${ }^{3}$, Joanne M. Devlin ${ }^{1}$, Gemma Vincent ${ }^{3}$, \\ Alexander Cameron", Stephen Graves ${ }^{3}$, Colin R. Wilks ${ }^{1}$ and Simon Firestone ${ }^{1}$
}

\begin{abstract}
Vaccination against Coxiella burnetii, the cause of $\mathrm{Q}$ fever, is reportedly the only feasible strategy of eradicating infection in ruminant herds. Preventive vaccination of seronegative goats is more effective in reducing shedding of C. burnetii than vaccinating seropositive goats. The age at which goats born on heavily-contaminated farms first seroconvert to $C$. burnetii has not yet been documented. In a 16-month birth cohort study, the age at which goats seroconverted against C. burnetii was investigated; 95 goats were bled every 2 weeks and tested for antibodies against C. burnetii. Risk factors for seroconversion were explored and goats shedding C. burnetii were identified by testing vaginal swabs taken at the goats' first kidding using a com1 polymerase chain reaction assay. The first surge in the number of goats with IgM to C. burnetii was observed at week 9 . Thus, a first vaccination not later than 8 weeks of age to control C. burnetii in highly contaminated environments is indicated. The odds of seroconversion were 2.0 times higher [95\% confidence interval (CI) 1.2, 3.5] in kids born by does with serological evidence of recent infection (IgM seropositive) compared to kids born by lgM seronegative does, suggesting either in utero transmission or peri-parturient infection. The rate of seroconversion was 4.5 times higher $(95 \% \mathrm{Cl} 2.1,9.8)$ during than outside the kidding season, highlighting the risk posed by $C$. burnetii shed during kidding, even to goats outside the kidding herd. Shedding of $C$. burnetii at kidding was detected in 15 out of 41 goats infected before breeding.
\end{abstract}

\section{Introduction}

Coxiella burnetii causes $\mathrm{Q}$ fever in humans, a disease that manifests with influenza-like symptoms including fever and pneumonia in approximately $30 \%$ of those acutely infected $[1,2]$. Chronic $\mathrm{Q}$ fever is characterised by debilitating arthritis, myopathy and cardiac malfunction [1-6]. Recent reports of chronic $\mathrm{Q}$ fever among individuals that had never been diagnosed with acute disease draws attention to the need for increased detection and treatment of sub-clinical Q fever infections $[1,2,7]$. The

\footnotetext{
*Correspondence: mmuleme@gmail.com;

michael.muleme@unimelb.edu.au

${ }^{1}$ Asia-Pacific Centre for Animal Health, Faculty of Veterinary

and Agricultural Sciences, The University of Melbourne, Parkville, VIC 3010,

Australia

Full list of author information is available at the end of the article
}

impact of the disease is further highlighted by increasing reports of chronic $\mathrm{Q}$ fever in children and mortalities of up to $13 \%$ among chronic $\mathrm{Q}$ fever patients [7-11]. These potentially severe impacts of $Q$ fever underscore the need to implement stringent prevention and control measures. It is therefore imperative to have evidence of how effective the different control measures are in the control of $C$. burnetii infections.

Many large $\mathrm{Q}$ fever outbreaks have been linked to farms with small ruminants and key control strategies have targeted reducing shedding of $C$. burnetii by the animals [12-15]. For example, a ban on breeding, culling of pregnant animals and vaccination of animals before breeding were used to control the large $\mathrm{Q}$ fever outbreak in the Netherlands [12-15]. Additionally, vaccination against $C$. burnetii in animals was shown to be more 
effective in reducing the shedding of the organism when carried-out in seronegative animals than in seropositive ones, underscoring the need to vaccinate animals before they are infected with C. burnetii [16-18].

The age at which most animals born on infected farms first seroconvert to $C$. burnetii has not been documented. Several previously published studies point to the possibility that goats get infected early in life. For example, during a human $\mathrm{Q}$ fever outbreak that was linked to a goat farm in France, 52\% of 3-4-month-old kids were reported to have been seropositive to $C$. burnetii; and approximately $90 \%$ of these were reported to have been infected with C. burnetii before they started kidding [19]. Similarly, 33\% (192/589) of kid goats in another study undertaken on a farm linked to a human $Q$ fever outbreak, were reported to have been infected before they started kidding and shed C. burnetii at their first kidding despite being kept away from adult goats as soon as they were born [17]. These studies do not indicate when the goats first seroconverted to $C$. burnetii although some of the results point to a time before 4 months of age.

Based on modelling of C. burnetii transmission and control in Dutch dairy farms, Bontje et al. found that vaccination of goats was the only control measure that could eradicate $C$. burnetii infections from infected herds [20]. The models estimated that it would take 7 years to eradicate the disease from infected farms if goats were to be vaccinated 1 month before breeding annually for all the 7 years [21]. There is a possibility that eradication of $C$. burnetii on infected farms could be achieved in shorter timeframes if vaccination of goats against $C$. burnetii were implemented at the age when most animals had not seroconverted. The Dutch transmission models supported the value of preventive vaccination over vaccination of already infected animals [20, 21]. Similarly, experimental and field studies that were carried out to assess the effectiveness of phase 1 vaccines administered prior to breeding mostly reported that vaccination only reduced the level of shedding at the herd level but did not prevent infection. This may be due to most of the goats being infected by the time of vaccination [16, 18, 19, 22].

This study therefore aimed to establish the age at which goats born on an intensively-managed goat dairy associated with human cases of $\mathrm{Q}$ fever first seroconverted to C. burnetii to provide recommendations on the timing of vaccination against $C$. burnetii. Additional objectives included: describing and comparing the nature (time of occurrence, duration and titres) of antibody-mediated immune responses to $C$. burnetii of goats that first seroconverted before the target breeding age ( 28 weeks) to those of goats that seroconverted later, and identifying risk factors and estimating any production losses associated with goats seroconverting before the target breeding age.

\section{Materials and methods}

\section{Study design}

In this longitudinal cohort study, 95 goats were followed from birth for 16 months. The animals were kept under routine animal management conditions on a large intensive dairy goat enterprise in Victoria, Australia. The enterprise holds approximately 5000 milking goats which are synchronised to concentrate kidding at four times each year (at March, June, September and November), in herds on five farms. The enterprise also has a flock of 1100 paddock-grazed extensively-managed dairy sheep. The sheep flock is kept separately from the goat flock. Since 2013, the enterprise was associated with 18 confirmed human cases of $\mathrm{Q}$ fever and tested polymerase chain reaction (PCR) positive for $C$. burnetii on various clinical and environmental samples [23]. Approximately 250 goats kid on each of the farms in every one of four kidding seasons, at a kidding rate of approximately two kids per goat. Only female kids and males from high producing animals (elite males) are kept for raising and production.

In herds infected with caprine arthritis and encephalitis virus (CAEV), some kids are routinely "snatch-reared", i.e. removed from their does before they feed on colostrum to prevent transmission of CAEV. These were then bottle-fed pooled colostrum from a CAEV negative farm. The 95 goats ( 90 female and 5 elite males) in this study were on two of the farms ("GS" and "LC"), both of which were CAEV-positive herds that were previously shown to have a high prevalence of C. burnetii [24]. Therefore, the kids in this study were mostly snatch-reared and were bottle-fed colostrum (from the herd on a third farm, "FH", holding CAEV-negative, but $C$. burnetii- positive goats) within $8 \mathrm{~h}$ after birth. The colostrum is routinely heattreated before it is fed to the goats. It is anticipated that heat-treatment would not lower the quality of immunoglobulins in the colostrum. The kid goats were then fed a milk replacer and weaned at approximately 10 weeks of age. At 28 weeks of age (target breeding age), the female goats that weighed $\geq 23 \mathrm{~kg}$ were mated using adult elite males (not the elite males followed in this study). The goats are routinely weighed at weaning and before breeding.

\section{Sample size}

The minimum required sample size was estimated to be 40 kids to have $80 \%$ power of detecting a statistically significant difference between paired samplings (day 0-2 weeks sampling as well as $0-12$ weeks) assuming seroconversion to $C$. burnetii would be very rare in kids between 0 and 2 weeks of age $(<1 \%)$ and that $20 \%$ of the kids would have seroconverted by 12 weeks of age; at a $5 \%$ chance of a type I error. Based on this sample size 
calculation, with consideration of anticipated mortality, losses to follow-up and multivariable effects, 95 goats were recruited from two kidding seasons; 33 in March 2014 kidding season (cohort 1) and 62 in June 2014 kidding season (cohort 2). Goats in cohort 1 were recruited from farms GS and LC while goats from cohort 2 were recruited only from LC. All animal sampling was conducted with the approval of the University of Melbourne Animal Ethics Committee, Application Number 1413118.

\section{Examination of antibody-mediated immune responses to $C$. burnetii in goats from birth to 16 months of age}

To investigate the age at which goats seroconvert to $C$. burnetii as well as to describe and compare antibodymediated immune responses to C. burnetii among goats that seroconverted before and after the target breeding age, blood samples were taken from the recruited goats at birth (before feeding colostrum), then every 2 weeks for the first 7 months of life and then every 4 weeks until the end of the study, and tested for serum IgM and IgG antibodies against $C$. burnetii antigens using a previously validated indirect immunofluorescence assay (IFA) [24].

In this study, the IFA applied was on a dilution series commencing at 1:160. As described in detail elsewhere [24], at this cut-off there was minimal background fluorescence in negative control goat samples obtained from New Zealand, an OIE-declared C. burnetii free country. Appling a 1:160 cut-off, the IFA has also been shown to be highly sensitive for IgG (94.8\%) and IgM antibodies $(88.8 \%)$ [24]. The repeatability of the IFA was previously estimated to be $100 \%$ for IgG phase $1,96.9 \%$ for IgG phase 2 and $78.1 \%$ for both IgM phase 2 and IgM phase 1 using samples re-tested after 3 months of storage at $4{ }^{\circ} \mathrm{C}$ [24]. The IFA slides were read by two experienced technicians with 94.4\% agreement (Cohen's Kappa $=0.88$ ) [24].

To quantify serum antibodies to C. burnetii two-fold serial dilutions of sera were prepared, from 1:160 (the previously validated cut-off value) to $1: 1280$, and tested for IgM and IgG antibodies against C. burnetii antigen using the IFA [24]. The testing of the twofold serial dilutions from 1:160 to 1:1280 was undertaken on sera from 54 of the 95 goats that were present throughout the first 12 months of the study. Among those excluded were animals that were lost to follow up, including those that died in the first 10 weeks of the study before immune responses were mounted. Repeat samples from each goat were also used to describe the duration of the immune response and the proportion of goats mounting secondary immune responses (immune responses that occur after the initial antibody response had declined). All samples were tested in duplicate for IgM and IgG against phase 1 and 2 C. burnetii.
Colostrum antibodies were identified through the occurrence of high IgG phase 1 titres in kid goats that were previously seronegative but seroconverted after feeding colostrum, as IgG antibodies occur late in the course of infection and are not expected to occur within 2 weeks in goats that had been negative. After the decay of IgG phase 1 maternal antibodies, new C. burnetii infections were considered to have occurred when serum IgM followed by IgG antibodies against phase 2 antigens were identified in previously seronegative goats or a twofold rise in antibody titre was detected following seroconversion to IgG phase 2 , as is routine practice in human and veterinary C. burnetii diagnostics $[25,26]$. Antibodies against the phase 2 antigens of $C$. burnetii appear early in the course of infection while antibodies against phase 1 antigens appear much later in the course of infection [25, 26]. Incidence rates were estimated using an animal time at risk denominator calculated as the number of weeks from birth to the sampling time immediately before the first seroconversion (from negative to positive for both IgM and IgG or $\geq$ twofold rise in IgG titre against phase 2) or loss to follow-up of each animal. Half of the time between the last negative sample and the first positive sample or loss to follow up was added to the length of time an animal was considered at risk [27].

Pooled colostrum collected from the CAEV-negative farm, FH, was tested for C. burnetii IgG antibodies to phases 1 and 2 C. burnetii using the IDEXX commercial ELISA kit validated for detecting antibodies in milk whey at a 1:5 dilution $[24,28]$. Undiluted whey from the pooled colostrum was also tested for IgG and IgM antibodies against phase 1 and 2 C. burnetii using the IFA protocol that had been validated for use with goat serum, to ascertain the type of antibodies the goats obtained from colostrum, as described previously [24, 28]. The colostrum was also tested for C. burnetii DNA using a DNA extraction protocol and PCR assay targeting the com 1 gene of $C$. burnetii as previously described [24, 29].

\section{Identification of risk factors associated with seroconversion to $C$. burnetii before breeding}

The probability of remaining seronegative at the different sampling points until the target breeding age was estimated using survival analysis. Initially, three parametric models (namely the Weibull, the Cox proportional hazard model and the Exponential model) were constructed to estimate the probability of goats remaining seronegative from birth to breeding. These were then analysed for goodness of fit based on the Akaike information criterion (AIC) [30]. As the Weibull model displayed the best fit to the data, the probability density function of the time to first seroconversion (change from negative to positive for both IgM and IgG or $\geq$ twofold rise in IgG titre against 
phase 2) $(t)$ was derived from the Weibull distribution using the equation:

$$
f(t)=\alpha \lambda t^{\alpha-1} e^{\lambda t^{\alpha}}
$$

where $f(t)$ is the probability density function of seroconversion at the different time points $t ; \lambda>0$ is the probability of seroconversion or scale parameter and $\alpha>0$ is the shape parameter. $\alpha$ is $1 / \sigma, \sigma$ being a variance-like parameter on the log-time scale. From this the survival (i.e. proportion yet to seroconvert at each timepoint), $S(t)$, was derived as:

$$
S(t)=e^{-\lambda t \alpha}
$$

The influence of qualitative risk factors including farm, cohort, C. burnetii exposure status of the source does, failure of passive transfer and the quality of passive transfer, on seroconversion was tested using the Weibull survival model. The $C$. burnetii exposure status of the source does was obtained by testing serum samples from the source does for IgG and IgM antibodies against $C$. burnetii at the time of birth of the study kid-goats. The occurrence of passive transfer was inferenced through the detection of IgG phase 1 maternally-derived antibodies in the study goats at 2 weeks of age while the quality of passive transfer was assessed using the titre of maternally-derived IgG phase 1 and 2 antibodies detected the kid-goats at 2 weeks of age as well as the detection of IgG phase 1 maternally-derived antibodies in kid goat sera after 4 weeks of age. The risk factors were included in separate univariable Weibull models to assess their influence on the probability of goats remaining seronegative or seroconverting at various times before the target breeding age. The univariable Weibull models testing the influence of risk factors on the probability of seroconversion is described by the equation:

$$
\lambda_{i}=e^{-\left(\mu+\beta x_{j}\right) / \sigma}
$$

where the influence of risk factor $x_{j}$, for the $i^{\text {th }}$ goat is modelled through the seroconversion probability parameter $\lambda_{\mathrm{j}}$ and $\alpha=1 / \sigma$.

Kaplan-Meier survival curves and univariable Weibull regression coefficients were then generated for different factors at cohort, farm (farm of birth), doe (IgG and IgM serological status) and individual kid level (level and duration of antibodies to C. burnetii derived from colostrum) and used to assess how the different risk factors influence seroconversion. Multivariable Weibull regression models were constructed (based on appropriate fit to the data) including all factors statistically significant in univariable analysis at $p<0.2$. The coefficients of the Weibull model were converted to hazard ratios using the ConvertWeibull package in $\mathrm{R}$ version 3.1 .3 [31, 32], and only variables associated with the relevant outcome at $p<0.05$ were retained in final models. The residuals and predicted values of the variables in the final models were analyzed and scaled-schoenfied residual plots were prepared for the variables in the final models.

To ascertain whether exposure to kidding periods resulted in increased numbers of infected goats, the rate of occurrences of IgM or IgG responses against C. burnetii during and outside the kidding season was compared using the animal time at risk denominator and the number of IgM and IgG responses as the numerator in the "compare 2 rates" function in Open Epi, version 3.01. The number of IgG and IgM responses were counted whenever a change from negative to positive to either IgM or IgG occurred during testing at the screening dilution. Primary and secondary IgM or IgG responses were considered to have occurred during the kidding period if they were detected within or $15 \mathrm{~d}$ after the kidding months. The extra $15 \mathrm{~d}$ were included to cater for the time required for antibody-mediated immune responses to occur in case goats became infected towards the end of the kidding season. Antibody-mediated immune responses that could have resulted from infections due to $C$. burnetii shed at either early kidding or late-term abortions, occurring within 2 weeks prior to the start of the kidding season, would fall within the kidding months and thus catered for in the "within kidding" counts of seroconversion. Only antibody-mediated immune responses that occurred after weaning (week 10) were included in this analysis as they were considered to be immune responses arising from environmental exposure to $C$. burnetii and not infection as neonates during the kidding process.

The period between any two consecutive sampling points from week 10 to the end of the study were classified as occurring "within" or "outside" the kidding season based on whether or not they fell during the kidding month and $15 \mathrm{~d}$ later. Animal time at risk "within" and "outside" the kidding period was computed for each animal as the number of weeks between the two consecutive sampling points if its samples tested negative at both points; half of the length of time between two sampling points if antibody-specific immune responses occurred (from negative to positive at 1:160 screening dilution), seroreversion (from a positive to either IgM or IgG at the 1:160 screening dilution to negative) or loss to follow-up occurred. For periods with one sampling point falling within the kidding season and the other outside the kidding season, the animal time at risk was divided equally between the within and outside kidding categories.

\section{Estimating the effect of pre-breeding seroconversions on shedding of $C$. burnetii at kidding and on production parameters}

To estimate the proportion of shedding arising from goats that seroconverted before the target breeding age, vaginal 
swabs were obtained within $24 \mathrm{~h}$ of the goats' first kidding and tested using the real genomics DNA extraction protocol (Real Biotech Company) and a PCR targeting the com1 gene of C. burnetii [29]. The difference in the risk of delayed kidding (not kidding by 12 months of age), delayed joining (not bred at 7 months) and shedding of C. burnetii among goats that seroconverted before the target breeding age and those that seroconverted after the target breeding age was estimated using two-by-two contingency tables, and comparing relative risks with estimation of $95 \%$ confidence intervals and Fisher's exact test statistic in STATA.

The association between seroconversion before breeding and outcomes of weight at weaning, and weight change between weaning and breeding, were estimated using multivariable linear regression models, and adjusting for cohort and farm, using STATA 13.0. The model residuals and the weight variables used were also analysed for normality in STATA 13.0.

\section{Results}

\section{Antibody-mediated immune responses to $C$. burnetii} in goats from birth to 16 months

No antibodies against $C$. burnetii were detected before goats were fed colostrum (Figure 1). High IgG antibody titres to phase 1 and phase $2 C$. burnetii were detected after feeding colostrum (Figure 1). IgG antibodies against phase $1 \mathrm{C}$. burnetii were used to differentiate maternally-derived antibodies from responses to C. burnetii exposure; IgG antibodies against phase 1 waned by week 7 (median duration of presumablycolostrum derived antibodies: 7 weeks; inter-quartile range: $4-7$ weeks). Of the 80 goats sampled after they had been fed colostrum, 71 (89\%) became seropositive for IgG to phase 1 antibodies (presumably maternallyderived). Furthermore, all these, except one, were also positive for IgG antibodies against phase 2 C. burnetii. Colostrum was tested and found to have IgG antibodies to phase 1 and 2 C. burnetii antigens using ELISA (optical density [OD] of 1.09 at 0.40 cut-off) and IFA (titres of 1024 IgG to phase 1, 1024 IgG phase 2) and contained negligible amounts of IgM against phase 2 (IFA titre $=40)$ and no IgM to phase 1 C. burnetii.

IgM seroconversion to phase 2 C. burnetii occurred as early as week 2 after birth; however, the first surge in the number of goats that seroconverted to phase 2 C. burnetii was observed at 9 weeks of age (Figure 2). Similarly, IgM titres to phase 2 C. burnetii rose as early as 2 weeks into the study, but the first peak was observed at 9 weeks of age as shown in Figure 1. This was followed by a rise in IgG titres to phase 2 C. burnetii and a rise in IgG titres to phase 1 C. burnetii at 24 and 40 weeks of age in cohort 2 and cohort 1 , respectively (Figure 1 ).
From birth to 10 weeks, 40 out of the 80 goats (50\%) present at the second sampling point showed IgM seroconversion to phase 2 C. burnetii while between week 10 and week 28 , another 17 out of the $76(22 \%)$ goats present at week 10 showed IgM seroconversion to phase 2 . From birth to week 10, IgG seroconversion to phase 2 was detected in 2 out of the 80 goats (3\%) and between week 10 and week 28 , a total of 38 out of the 76 goats (50\%) showed IgG seroconversion to phase 2 . One goat had IgG antibodies against phase 2 without a detectable IgM response against phase 2 .

Nine goats did not have detectable IgG antibodies to phase 1 after feeding colostrum. Of these, seven goats showed IgM seroconversion to phase 2 by week 28 (three at week 2, three at week 7 and one at week 25) and only one seroconverted after week 28 . One of the nine goats that did not have IgG to phase 1 after feeding colostrum died at week 4 before seroconverting to C. burnetii.

A total of 19 out of the 95 goats died by week 5 , before any seroconversion was observed. Of these, only three had the initial IgG to phase 1 and 2 observed after feeding colostrum while one goat did not have IgG antibodies to phase 1 after feeding colostrum. The rest of these goats (15) died before the second sampling, thus their serological status after feeding colostrum could not be assessed. Another six goats died between week 5 and week 10, all of which had detectable IgG to phase 1 after feeding colostrum. One of these showed IgM seroconversions to phase 2 . An additional eight deaths occurred between week 10 and week 28 , all except one had IgG antibodies against phase 1 after feeding colostrum and only three had seroconverted with IgM against phase 2 .

The crude incidence rate was seven seroconversions $(95 \%$ CI $5.7,8.9)$ per 100 goat weeks at risk. Equivalent interpretations include seven seroconversions per 100 goats at risk for 1 week, or per 50 goats followed for 2 weeks. Whilst there was no statistically significant difference in the rates when compared across time periods (from birth to weaning, weaning to target breeding age and after the target breeding age), see Table 1, there was a trend suggesting gradually increasing risk over time.

Secondary IgM against phase 2 was detected in 42 out of the 70 goats $(60 \%)$ present at the time of weaning. A total of 24 of these secondary IgM immune responses occurred before the target breeding age and the other 18 occurred after the target breeding age. Secondary IgG immune responses against phase 2 were detected in only 11 goats, four of these occurring before the target breeding age. Only seven goats had a secondary IgM response against phase 1 and only one goat had a secondary IgG response against phase 1 . 


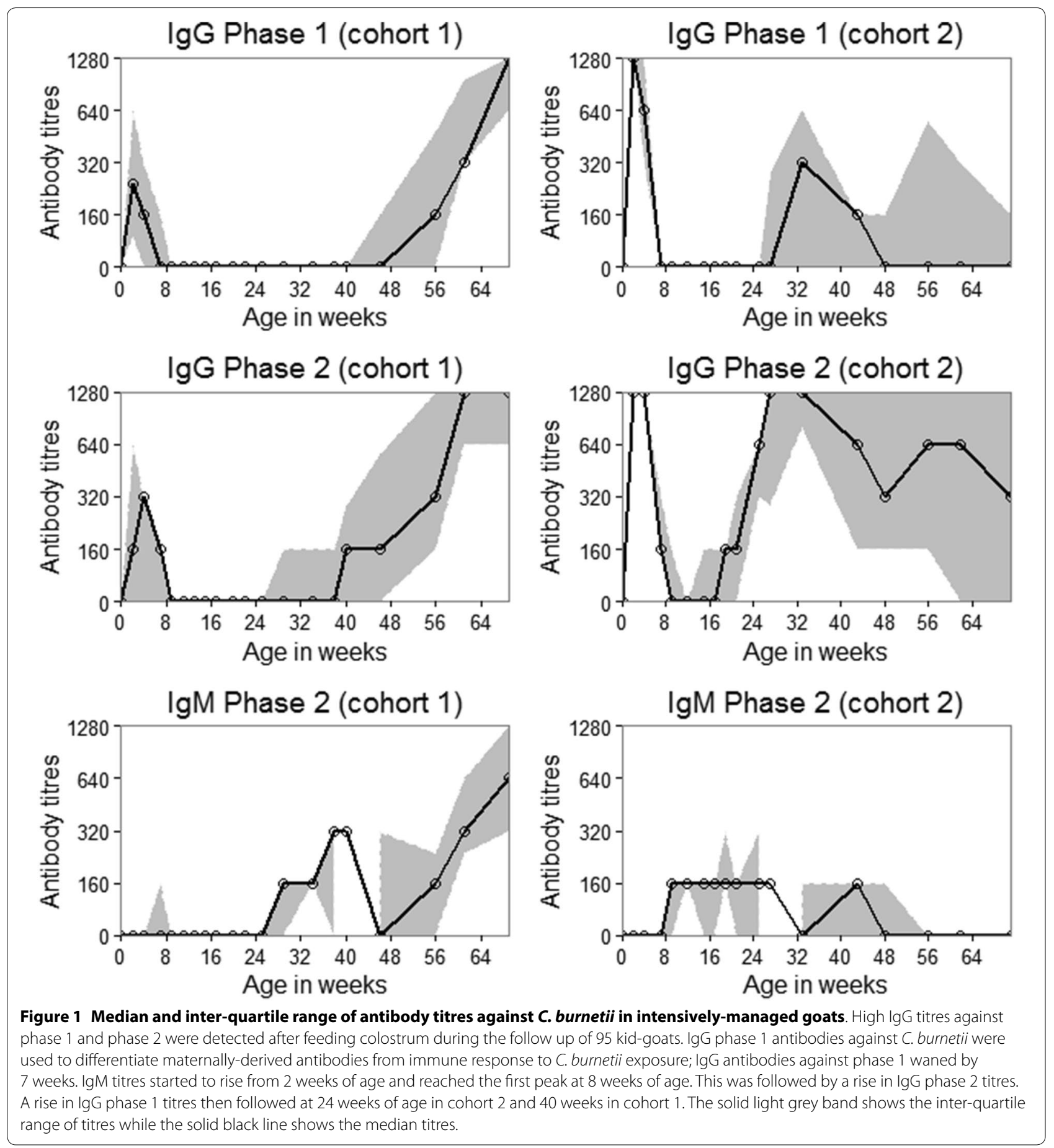

The duration of IgG and IgM immune responses are described in Table 2. The duration of primary immune responses was significantly higher in cohort 2 than in cohort 1, except for IgM against phase 2 as shown in Figure 3. Overall, immune responses that occurred before the target breeding age were not statistically different from those that occurred after the target breeding age (Table 2).
Risk factors associated with seroconversion to C. burnetii before breeding

The probability of goats remaining seronegative to $C$. burnetii for the first 28 weeks of life was 16.7\% (95\% CI 9.4, 29.4) (Additional file 1). The Weibull model had the best fit for these data with (AIC $=440.9)$ compared to the Cox proportional hazards model $(\mathrm{AIC}=457.5)$ and the 


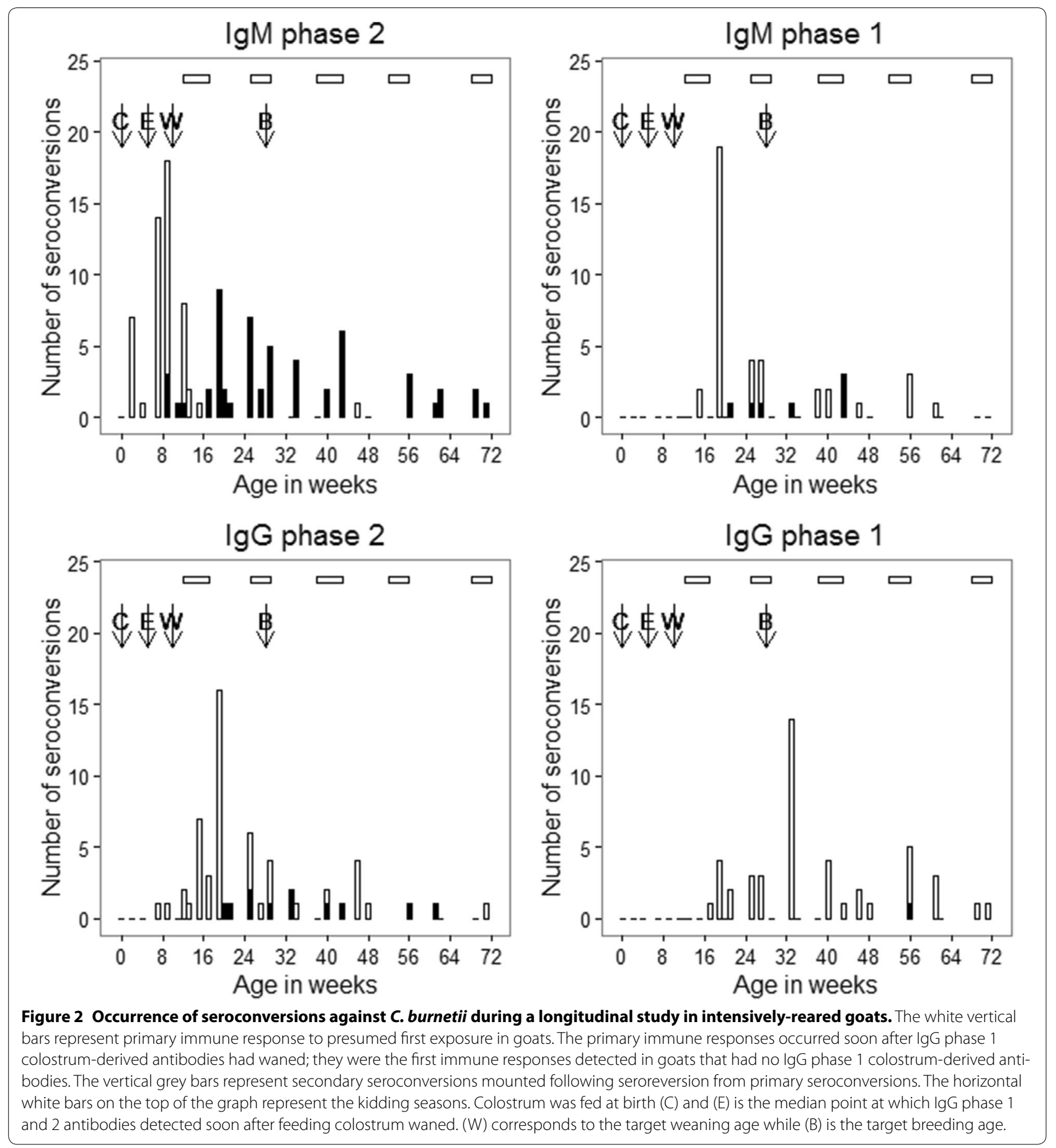

Exponential model $(\mathrm{AIC}=460.6)$. Being from farm GS, being a member of cohort 1, having colostrum-derived IgG phase 1 antibodies titres $\geq 320$ and being born by an IgM seronegative doe were all associated with a higher probability of not seroconverting against $C$. burnetii before 28 weeks of age on univariate analysis as shown in Figure 4. Outputs of multivariable Weibull regression are presented in Table 3, and for completeness, univariable results are presented in Additional file 2. The odds of seroconversion were 2.0 times higher [95\% confidence interval (CI) 1.2, 3.5] in kids born by does with serological evidence of recent infection (IgM seropositive) compared to kids born by IgM seronegative does. Model residual analysis showed that the assumptions for the Weibull model were not violated. 
Table 1 The incidence rate of seroconversion to $C$. burnetii before and after the breeding age.

\begin{tabular}{|c|c|c|c|c|c|}
\hline Time period (weeks) & Number at risk & Goat weeks at risk & $\begin{array}{l}\text { Number of seroconver- } \\
\text { sions }\end{array}$ & $\begin{array}{l}\text { Incidence rate (per } 100 \\
\text { goat weeks at risk) }\end{array}$ & $\begin{array}{l}\text { Incidence rate ratio }(95 \% \\
\mathrm{Cl})\end{array}$ \\
\hline $0-10$ & 95 & 618.5 & 40 & $6.5(4.6,8.8)$ & 1.00 (reference) \\
\hline $11-28$ & 32 & 238.0 & 18 & $7.6(4.5,12.0)$ & $1.17(0.67,2.04)$ \\
\hline $29-71$ & 8 & 61.5 & 8 & $13.0(5.6,25.6)$ & $2.01(0.94,4.30)$ \\
\hline Total & 135 & 918.0 & 64 & $7.0(5.7,8.9)$ & - \\
\hline
\end{tabular}

10 weeks is the target weaning age, 28 weeks was the target breeding age in the intensively-managed goats on the study farm in Victoria, Australia, $2014-2015$ Cl confidence interval

Table 2 Comparison of the duration of pre-breeding to post-breeding antibody responses to C. burnetii in goats.

\begin{tabular}{|c|c|c|c|c|c|c|}
\hline \multirow[t]{2}{*}{ Antibody type } & \multirow[t]{2}{*}{ Type of immune response } & \multirow[t]{2}{*}{ Time of occurrence } & \multicolumn{4}{|c|}{ Duration of antibody responses in weeks } \\
\hline & & & n (rev) & Mean & Median (range) & $p$ value* \\
\hline \multirow[t]{4}{*}{ IgM phase 2} & Primary & Pre-breeding & $57(55)$ & 10.9 & $5.5(1.0,51.0)$ & 0.570 \\
\hline & & Post-breeding & $7(7)$ & 5.9 & $6.0(2.0,12.0)$ & \\
\hline & Secondary & Pre-breeding & $24(24)$ & 10.4 & $9.0(1.0,34.0)$ & 0.523 \\
\hline & & Post-breeding & $18(18)$ & 8.4 & $4.0(2.0,33.0)$ & \\
\hline \multirow[t]{4}{*}{ IgG phase 2} & Primary & Pre-breeding & $40(27)$ & 19.4 & $12.0(1.0,48.5)$ & 0.376 \\
\hline & & Post-breeding & $14(5)$ & 7.5 & $6.0(2.5,13.0)$ & \\
\hline & Secondary & Pre-breeding & $4(3)$ & 30.7 & $42.5(3.0,46.5)$ & 0.564 \\
\hline & & Post-breeding & $7(2)$ & 25.8 & $25.8(17.0,34.5)$ & \\
\hline \multirow[t]{4}{*}{ IgM phase 1} & Primary & Pre-breeding & $29(29)$ & 5.1 & $4.0(1.0,12.0)$ & 0.197 \\
\hline & & Post-breeding & $9(7)$ & 2.9 & $3.0(1.0,6.0)$ & \\
\hline & Secondary & Pre-breeding & $3(3)$ & 1.3 & $1.0(1.0,2.0)$ & 0.127 \\
\hline & & Post-breeding & $4(4)$ & 2.9 & $3.5(1.0,3.5)$ & \\
\hline \multirow[t]{4}{*}{ lgG phase 1} & Primary & Pre-breeding & $40(12)$ & 23.7 & $22.5(5.0,42.5)$ & 0.897 \\
\hline & & Post-breeding & $14(14)$ & 20.5 & $22.3(5.0,34.5)$ & \\
\hline & Secondary & Pre-breeding & $0(0)$ & - & - & - \\
\hline & & Post-breeding & $1(1)$ & 11.5 & - & \\
\hline
\end{tabular}

Pre-breeding responses are those that started before the target breeding age while post-breeding responses are those that started after the target breeding age in intensively managed goats on the study farm in Victoria, Australia, 2014-2015

rev. number of antibody responses that seroreverted (moved from positive to negative) which were used in calculating the duration of serological positivity, $n$ total number of antibody responses

${ }^{*} p$ values derived using the Mann-Whitney $\mathrm{U}$ test comparing the duration of pre-breeding immune responses to the post-breeding immune responses

The rate of occurrence of IgG immune responses to $C$. burnetii was 4.5 times higher $(95 \%$ CI 2.1, 9.8) within the kidding season than outside the kidding season among goats in cohort 2 (Additional file 4), whereas, the rate of occurrence of IgG immune responses was comparable within and outside the kidding season among goats in cohort 1 .

The effect of pre-breeding seroconversions on the shedding of $C$. burnetii and on production parameters Overall, 18 out of 46 goats were detected as shedding C. burnetii by PCR at their first kidding. Shedding was detected in 15 out of the 41 goats that seroconverted before week 28, some of which remained seropositive until kidding (Additional file 5). Out of the five goats that had not seroconverted by week 28 , three goats had detectable C. burnetii DNA in vaginal swabs taken at their first kidding.

The proportion of goats that were joined on time was comparable in goats that seroconverted before 28 weeks (28/50 goats, $56.0 \%)$ and those that had not seroconverted by week 28 (6/8 goats, $75.0 \%)$. Similarly, the proportion of goats kidding on time was comparable in goats that seroconverted before week 28 (19/50 goats, 38.0\%) and those that had not seroconverted by week $28(5 / 8$ goats, $62.5 \%$ ).

The multivariable linear regression model showed no statistically significant difference in pre-breeding weight between goats that seroconverted early and those that seroconverted later (Table 4). At weaning, goats in cohort 


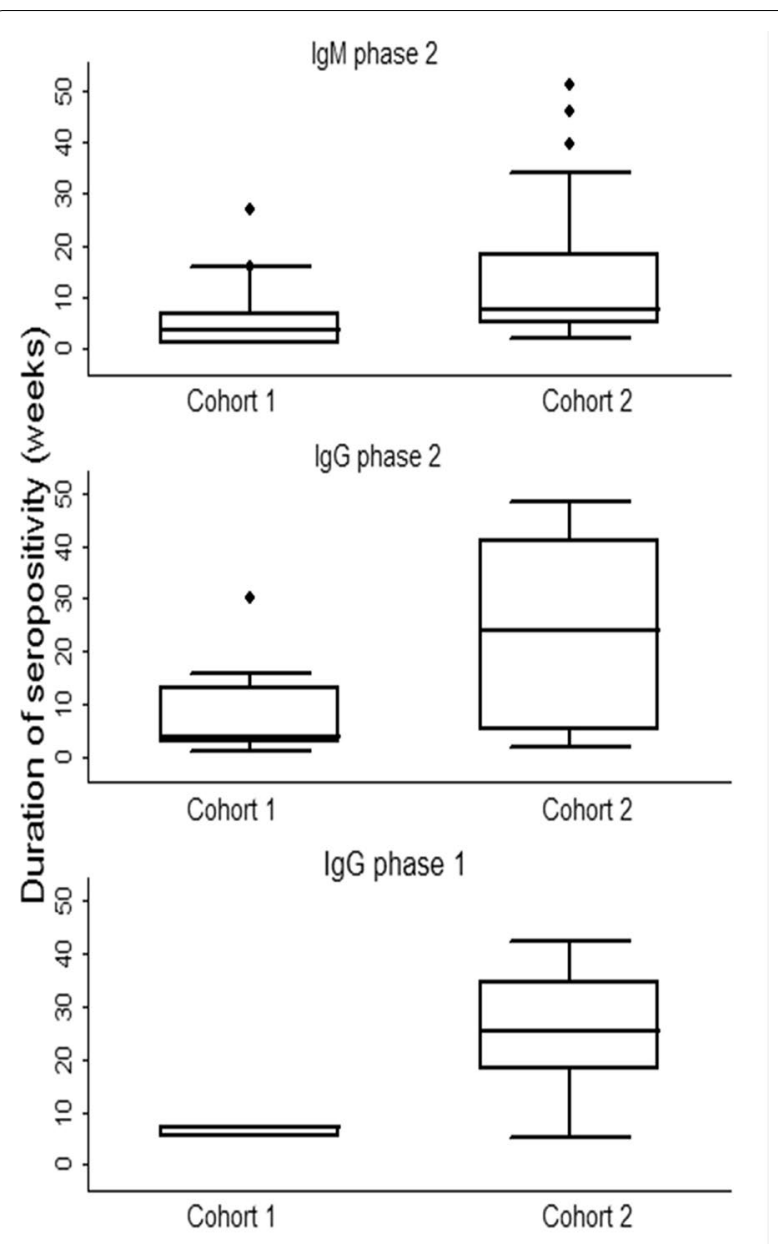

Figure 3 Box plots of the duration of $\lg G$ and $\lg M$ antibodies against $\boldsymbol{C}$. burnetii in intensively-reared goats. Overall the duration of antibodies to C. burnetii was higher in cohort 2 compared to cohort 1.

2 weighed $4.8 \mathrm{~kg}$ less than goats in cohort 1 (95\% CI 6.6, $3.1 \mathrm{~kg}$ less); and 30 out of the 49 goats (61\%) with weaning weight records in cohort 2 had seroconverted to either $\operatorname{IgG}$ or IgM phase 2 by week 10 while only 5 out of the 13 of goats (39\%) with weaning weight data in cohort 1 had seroconverted by week 10 . Between the time of weaning and the target breeding age, goats in cohort 2 gained $5.3 \mathrm{~kg}$ more than goats in cohort 1 (95\% CI $2.4,8.1)$. Only 10 out of the 38 goats (26\%) in cohort 2 seroconverted between weaning and the target breeding age compared to 6 out of the 12 goats $(50 \%)$ in cohort 1 . The distribution of the model residues and weight variables used in the model were within acceptable limits (skewedness \pm 1 ).

\section{Discussion}

To the best of our knowledge, this is the first birth cohort study to systematically investigate $C$. burnetii seroconversions in goats on infected farms over an extended period from birth. Furthermore, this study was undertaken in the absence of vaccination of goats or other control measures that would reduce the shedding of $C$. burnetii in infected goats. Therefore, the patterns of seroconversion or transmission described in this study can be considered representative of transmission of $C$. burnetii among young goats in a heavily contaminated intensive dairy herd.

Detection of antibodies in serum was considered the most effective way of detecting recent infection with $C$. burnetii in goats before breeding. Substantial quantities of $C$. burnetii are shed in milk, vaginal mucus and faeces around parturition due to massive replication of $C$. burnetii in the placenta [33-35]. However, shedding is only detectable over a very short timeframe and not before breeding [33-35]. Additionally, C. burnetii is present in low concentrations in blood, and for only a few days following infection, unlike antibodies which appear 1-3 weeks after infection and typically last for weeks to months depending on antibody class [26].

High proportions of goats had seroconverted to IgM and IgG against phase 2 C. burnetii by week 28 of the study which indicates that majority of the goats born on C. burnetii positive farms are infected early in life. Seroconversions were observed well before the goats were mated, which is contrary to the notion that trophoblasts are required for establishment of C. burnetii infection in ruminants. Infection of young non-pregnant goats with C. burnetii has been disputed in previous studies [17, 19, 36-39]. Although some studies suggest that C. burnetii requires trophoblasts for successful establishment of infection in ruminants $[35,36,40,41]$, we are not aware of any study that has investigated whether the absence of trophoblasts in young and male animals prevented the establishment of $C$. burnetii infection. Previous work has suggested that $C$. burnetii infections require trophoblasts to infect ruminants. This was hypothesised after observing primary replication and histopathological lesions only in trophoblasts following experimental infection of pregnant goats with C. burnetii [36]; that finding has been referenced in other studies [20]. However, it is possible that the organisms could have been present in other tissues in quantities undetectable by the methods used, as noted by the authors of the study [36].

Despite some studies suggesting that trophoblasts are required for establishing infection in ruminants, a number of studies investigating other aspects of $C$. burnetii on infected farms have reported a high proportion (> 50\%) of seroconversions to $C$. burnetii in young ruminants before breeding $[17,19]$, which is similar to the level detected in our study. Another study reported a seroprevalence of $9.8 \%$ in 6-month-old goats that had been kept in a closed facility with no exposure to adult goats [39]. In 


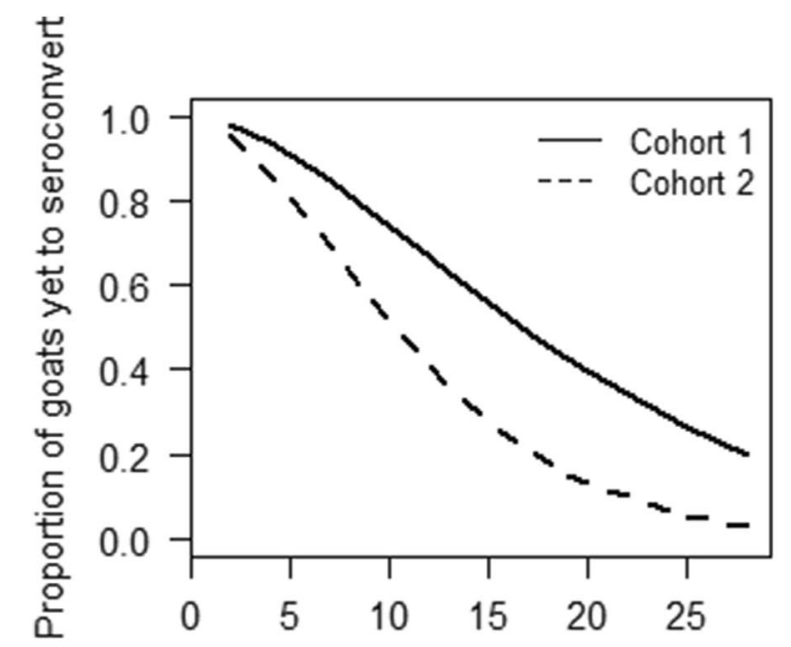

Age in weeks

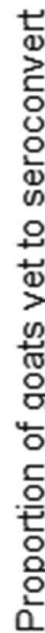

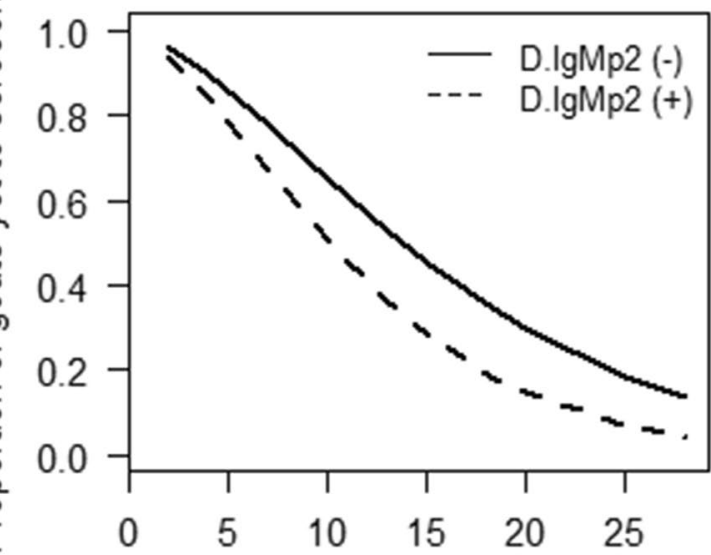

Age in weeks
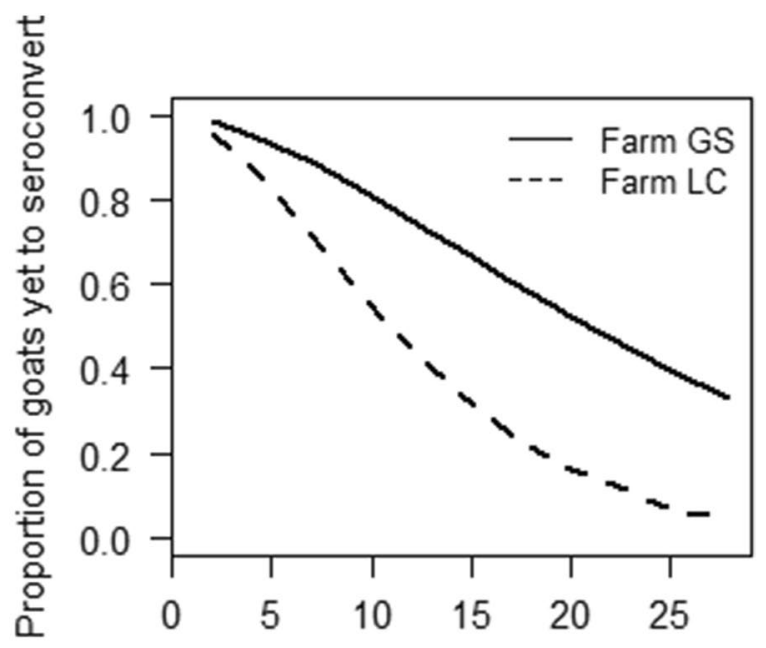

Age in weeks

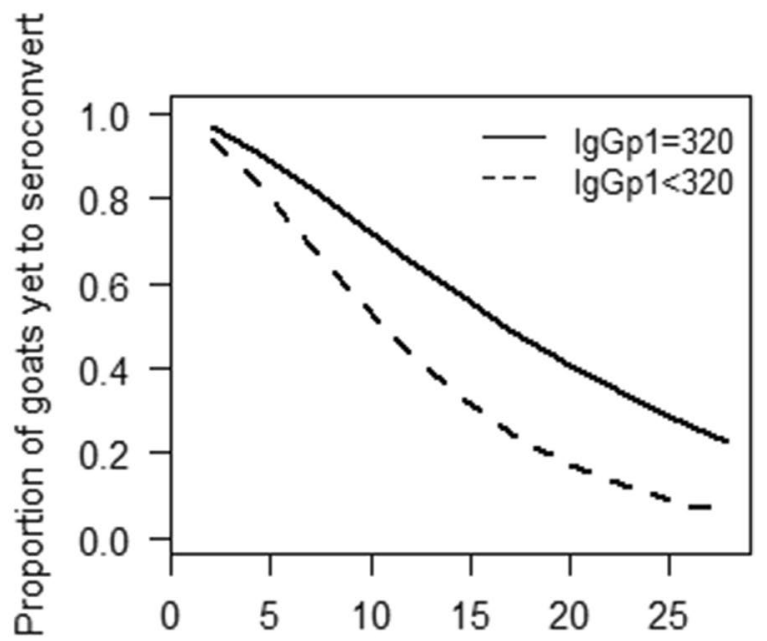

Age in weeks

Figure 4 Weibull survival curves comparing the pre-breeding probability of remaining seronegative to $C$. burnetii in goats.

D.lgMp2 = IgM serological status of the source does with (D.IgMp2(-) representing negative does and (D.IgMp2(+) the positive ones. IgGp1 =colostrum derived antibody titres dichotomised at a cut-off of 320 (IgGp1). Goats in cohort 1, farm GS and those born by (D.IgMp2(-) as well as those with $\lg \mathrm{Gp} 1=320$ had statistically significantly $(p<0.1)$ higher probabilities of remaining seronegative before breeding than goats from cohort 2, farm LC as well as those from D.IgMp2(+) and those with $\operatorname{lgGp} 1<320$ colostrum antibodies.

some instances, young animals were thought not to pose much risk in the transmission of $C$. burnetii thus excluding them from C. burnetii studies [16]. It is likely that the discrepancy in the proportion of young animals that seroconvert to C. burnetii among several studies [17, 19, 39 ] is highly dependent on the proportion of parent does shedding C. burnetii and the timing of the samplings relative to the progression of the outbreak. The proportion of adult goats shedding C. burnetii is likely to increase over time in an uncontrolled outbreak thus increasing the dose of $C$. burnetii organisms each susceptible animal in the herd is exposed to; and henceforth increasing the number of animals infected with C. burnetii. It is not clear if the build-up in the proportion of goats shedding C. burnetii would be affected by development of immunity after repeated exposure to $C$. burnetii but one study 
Table 3 Multivariable Weibull accelerated failure-time regression model assessing risk factors for seroconversion to $C$. burnetii before breeding.

\begin{tabular}{lllllllll}
\hline Variable & Levels & $\mathbf{n}$ & Serocon. & Coef. & SE (Coef.) & p value & Survival rate ratio (95\% Cl) & Hazard ratio (95\% Cl) \\
\hline Cohort & Cohort 2 & 62 & 42 & -0.60 & 0.11 & 0.001 & $0.55(0.39,0.78)$ & $2.73(1.45,5.16)$ \\
& Cohort 1 & 33 & 16 & 0.00 (ref) & & & 1.00 & 1.00 \\
Doe lgM & Positive & 37 & 29 & -0.42 & 0.16 & 0.008 & $0.65(0.48,0.89)$ & $2.04(1.19,3.54)$ \\
& Negative & 56 & 28 & 0.00 (ref) & & & 1.00 & 1.00 \\
Intercept & - & - & - & 3.89 & 0.35 & $<0.001$ & - & - \\
\hline
\end{tabular}

In intensively-reared goats in Victoria, Australia, 2014-2015. Interpretation: After adjusting for the effect of cohort, kids born by does that had positive lgM titres (Doe $\lg M=$ positive; indicating recent exposure) were 2.04 times more likely to seroconvert within the first 6 months of life compared to those born by lgM seronegative does. Log likelihood = -201.1. Coef. coefficient. Similarly, after adjusting for the effect of farm, kids born by does that had positive lgM titres were 2.23 times (95\% $\mathrm{Cl}$ $1.29,3.86$ ) more likely to seroconvert within the first 6 months of life compared to those born by lgM seronegative does; see Additional file 3

Table 4 Multivariable linear regression analysis of the effect of seroconversions to C. burnetii on goat weights.

\begin{tabular}{|c|c|c|c|c|c|c|c|}
\hline Model & Variable & Levels & $\mathrm{n}$ & Coef. & SE (coef.) & $p$ value & $95 \% \mathrm{Cl}$ of Coef. \\
\hline \multirow[t]{8}{*}{ Effect of seroconversion on weight at weaning ${ }^{a}$} & \multirow[t]{2}{*}{ Cohort } & Cohort 2 & 49 & -4.84 & \multirow{2}{*}{0.88} & \multirow[t]{2}{*}{$<0.001$} & \multirow[t]{2}{*}{$-6.61,-3.08$} \\
\hline & & Cohort 1 & 13 & 0.00 (ref) & & & \\
\hline & \multirow[t]{2}{*}{ Sex } & Male & 4 & 3.64 & \multirow{2}{*}{1.46} & \multirow[t]{2}{*}{0.016} & \multirow[t]{2}{*}{$0.71,6.56$} \\
\hline & & Female & 58 & 0.00 (ref) & & & \\
\hline & \multirow[t]{3}{*}{ Time of first seroconversion } & After 28 weeks & 10 & -0.33 & 1.00 & 0.740 & $-1.03,2.35$ \\
\hline & & 10-28 weeks & 17 & 0.66 & \multirow{2}{*}{0.84} & \multirow[t]{2}{*}{0.435} & \multirow[t]{2}{*}{$-2.36,1.66$} \\
\hline & & 0-10 weeks & 35 & 0.00 (ref) & & & \\
\hline & Intercept & - & - & 17.71 & 0.89 & $<0.001$ & $15.93,19.48$ \\
\hline \multirow{6}{*}{$\begin{array}{l}\text { Effect of seroconversion on weaning to breeding } \\
\text { weight change }^{b}\end{array}$} & \multirow[t]{2}{*}{ Cohort } & Cohort 2 & 38 & 5.27 & \multirow[t]{2}{*}{1.41} & \multirow[t]{2}{*}{0.001} & \multirow[t]{2}{*}{$2.43,8.11$} \\
\hline & & Cohort 1 & 12 & 0.00 (ref) & & & \\
\hline & \multirow[t]{3}{*}{ Time of first seroconversion } & After 28 weeks & 3 & 0.98 & 2.52 & 0.700 & $-4.09,6.04$ \\
\hline & & 10-28 weeks & 16 & -0.60 & \multirow{2}{*}{1.31} & \multirow[t]{2}{*}{0.652} & \multirow[t]{2}{*}{$-3.23,2.04$} \\
\hline & & 0-10 weeks & 31 & 0.00 (ref) & & & \\
\hline & Intercept & - & - & 7.67 & 1.40 & $<0.001$ & $4.86,10.49$ \\
\hline
\end{tabular}

Interpretation: ${ }^{a}$ No statistically significant difference in weaning weight was observed between goats that seroconverted before breeding and those that seroconverted post-breeding after adjusting for cohort and sex. However, goats in cohort 2 had weighed $4.84 \mathrm{~kg}$ lower at weaning than goats in cohort 1

b Similarly, no statistically significant difference in weaning-breeding weight change was observed between goats that seroconverted before breeding and those that seroconverted post-breeding after adjusting for cohort. Surprisingly, goats in cohort 2 weighed $5.27 \mathrm{~kg}$ more than goats that had not seroconverted by breeding. Sex was not included in model ${ }^{b}$ because all male animals were lost from the study by breeding time

reported that infected goats shed $C$. burnetii for at least two successive kiddings [34].

Furthermore, studies that detected C. burnetii organisms in spleens, liver, lungs, kidneys and hearts of young goats [18, 36, 37, 42] as well as interstitial non-suppurative pneumonia and granulomatous hepatitis lesions, similar to those observed in adult goats infected with $C$. burnetii, adds further evidence to the notion that goats can be infected early in life [42]. In most of these studies, it is apparent that diagnosis was made on a few tissues that were submitted, and the majority of the tissues were from kids that died perinatally as well as those that had been sacrificed after birth.

These studies provide useful clues to potential sites of replication and persistence of $C$. burnetii infection in non-pregnant young goats that could possibly result in shedding of low levels of the bacterium before breeding. However, the site of persistent $C$. burnetii infection in young ruminants is still unknown, unlike in adult goats where $C$. burnetii infection persists in the mammary glands and uterus resulting in continuous shedding of C. burnetii in milk long after kidding $[43,44]$. The site of persistent infection in young animals needs to be investigated, notwithstanding the possibility that the organism may be present in low numbers below the detection limit of most diagnostic tools.

The initial rise in IgG phase 1 and IgG phase 2 following colostrum feeding is probably due to uptake of antibodies against $C$. burnettii in colostrum. This is further supported by the high concentrations of IgG specific for phase 1 and phase 2 as well as negligible quantities of IgM that were detected in colostrum. The IgG and IgM 
concentrations in colostrum reported by this study are in accordance with another study that reported colostrum IgG concentrations of $41.2 \mathrm{mg} / \mathrm{mL}$ and IgM concentrations of $1.9 \mathrm{mg} / \mathrm{mL}$ [45]. In the event of infection at birth or around the time of feeding colostrum, IgG antibodies to phase 1 against such infections would not be detectable within 2 weeks as IgG phase 1 antibodies appear much later in the course of infection [26, 36, 46, 47]. Thus, the extremely high IgG phase 1 and phase 2 antibody titres (Figure 2) observed in the serum of kids at 2 weeks were most likely derived from colostrum.

Early IgM responses were detected mostly in kids that did not receive sufficient colostrum. This suggests that antibodies and other immune components in colostrum are protective against $C$. burnetii infection. This further explains the surge in IgM and IgG immune responses after the IgG phase 1 colostrum antibodies (antibodies detected immediately after feeding colostrum) wane, around week 7 (median), and thus suggests that goats are at risk after the depletion of colostrum antibodies. These findings also suggest that, in this study, ingestion of colostrum was protective in goat kids.

Colostrum could also have been a source of C. burnetii infection although this appears not to have been the case in this study possibly due to heat treatment of colostrum before it is fed to the goats. In any case, it appears that infection resulting from ingestion of contaminated milk is not readily achieved and observations in humans ingesting contaminated milk failed to demonstrate resulting infection $[48,49]$. It is therefore worthwhile to ensure that all goats are fed enough colostrum containing antibodies against $C$. burnetii, especially in instances where new-born goats are snatched from their mothers before they ingest colostrum. Perhaps, efforts could also be aimed towards increasing antibodies and immune components against $C$. burnetii in colostrum indirectly by giving a booster vaccination against $C$. burnetii to pregnant goats, as demonstrated in a vaccination trial of sows against porcine circovirus type 2 in pigs where the aim was to protect piglets from infection with this virus early in life [50]. Also, studies in which, pregnant animals were vaccinated using phase $1 \mathrm{C}$. burnetii vaccines have not reported any deleterious effects of the vaccine $[22,36]$.

In a dairy farm setting where new-born animals are restricted from receiving milk from their does, it seems reasonable to protect these animals as early as possible. However, considering the workload on intensive dairy farms during and immediately after kidding and the difficulty of ensuring that all kids receive adequate colostrum intake, we recommend vaccination be implemented not later than 8 weeks of age, i.e. preceding the majority of seroconversions in this study. We also recommend a booster dose to increase vaccine coverage, considering that antibodies derived from colostrum may still be present in some goats at 8 weeks of age and interfere with antibody production, even for inactivated vaccines like the Coxevac vaccine, (CEVA, France) used to vaccinate against $C$. burnetii in goats [51].

Cell-mediated immunity also plays an important role in protection against intra-cellular pathogens like $C$. burnetii and it is not affected by the presence of maternally derived antibodies, as demonstrated in a study that evaluated vaccination against Aujesky's disease in pigs [51-53]; early vaccination, even in the presence of maternal antibody, may still be protective. Some studies have however, shown that some components of the immune system, for example, the B cells derived from the intestinal lymphoid tissues are not produced until after 8 weeks of age in goats [54]. Ideally the best time to vaccinate the kids would be during the narrow window of opportunity that occurs between the decline of the colostrum-derived maternal antibodies and the synthesis of kid-endogenous antibody arising from their environmental exposure to $C$. burnetii. This is probably from 8 to 10 weeks of age.

The comparable duration of seropositivity and median antibody titres of goats that seroconverted prior to and after the target breeding age suggests similarity in exposure before and after breeding. The duration of seropositivity varied widely, which accords with what has been previously reported in another outbreak in the UK [39]. The finding that the IgG phase 2 and 1 responses last longer than the IgM phase 2 and 1 responses that precede them is consistent with what has been reported in human serology and in adult animal studies $[26,46]$. However, the number of secondary IgM responses compared to IgG responses, described in Table 2, may have been confounded by the lower repeatability of the IFA for IgM (78.1\%) compared to IgG antibodies $(96.9 \%)$ as well as the lower sensitivity of the IFA for IgM (88.8\%) compared to IgG antibodies (94.8\%).

The difference in risk associated with the two cohorts from two different kidding times studied here suggests that differences in the level of $C$. burnetii shedding at each kidding season, and possibly different levels of exposure to C. burnetii at birth, or soon after, may influence timing of occurrence of seroconversions against $C$. burnetii infections. This could also indicate differences in colostrum quality and its administration. Similarly, the surge in IgM and IgG seroconversions that occurred prior to weaning could possibly be due to exposure to $C$. burnetii at birth. The difference in the risk of seroconversion at farm level points to variability in contamination or shedding patterns on the different farms on the property; animals on Farm GS were partly reared by less 
intensive small out-grower farms until 4 months of age, which may explain the lower risk of seroconversion compared to Farm LC.

The increased risk of seroconversion in kids born to IgM seropositive does suggests recent infections in these does and points to either in utero transmission of C. burnetii or periparturient transmission. In utero transmission of $C$. burnetii has not been confirmed but C. burnetii DNA has been detected in amniotic fluid as well as spleen and kidneys of live and aborted kids; histopathological lesions similar to those in infected adult goats have also been reported in new-born goats [18, 36, 38]. Another study has also shown goat embryos to be highly susceptible to C. burnetii infection in vitro [55]. However, there is a challenge in differentiating DNA present because of $C$. burnetii infection from that due to contamination from the heavily infected placentas. The majority of the reports of PCR positive results from tissues of aborted kids provided very little information on how the prevention of contamination of these tissues by DNA from the heavily infected placental tissues was achieved [18, 36, 37]. Perhaps, the demonstration of vertical transmission of $C$. burnetii using PCR needs to be complemented by other methods, such as immunohistochemistry, that detect $C$. burnetii within the cells in tissues of foetuses or newborn kid goats.

As expected, exposure to $C$. burnetii shed at subsequent kidding seasons could be playing a role in increasing the proportion of infected goats before the target breeding age. This was more evident in cohort 2 , where the rate of occurrence of antibody mediated responses was 4.5 times higher within than outside the kidding season. The comparable rate of occurrence of immune responses within and outside the kidding season for cohort 1 could be a result of a proportion of these goats being reared on out-grower farms for the first 4 months of their lives. The occurrence antibody-mediated immune responses outside the kidding seasons points to other risks of exposure to C. burnetii in the environment; for example, contaminated straw, hay or pastures, as well as inhalation or ingestion of contaminated dust particles especially during dry and windy weather [56-58].

Although occurrence of IgM and IgG responses and not the conventional twofold rise in IgG against phase 2 or IgM followed by IgG were used in comparing outside and within kidding season exposure to C. burnetii, this seemed the most practical way of computing animaltime at risk given the shorter duration of IgM antibodies compared to IgG as shown in Table 2. Comparisons for within and outside kidding seasons were also restricted to either IgM and IgG to cater for the differences in duration of IgM and IgG seropositivity as well as the differences in repeatability and sensitivity of the IFA for IgM and IgG antibodies. The detection of antibodies using the IFA may have been enhanced by repeated sampling and testing of samples in duplicate against IgG and IgM to phase 1 and 2 C. burnetii given that all IgG responses except one were preceded by IgM responses. The performance of the IFA may also have been enhanced by the quality of antigens used and the experience of the technicians in this instance, as these factors may influence the reproducibility of the test.

The shedding of C. burnetii by goats that seroconverted before breeding and remained seropositive through pregnancy underscores the role played by pre-breeding infections in the transmission of infection within the herd. Owing to mortality, we did not have a sufficiently large sample size at the end of the study to detect any statistically significant differences in C. burnetii shedding patterns between goats that seroconverted before breeding and those that seroconverted after breeding. Similarly, we were not able to detect any statistically significant reductions in weight gain at the individual animal level. These considerations are the focus of further ongoing studies. The high mortalities reaching up to $20 \%$ in the first months of follow-up were thought to result from a number of factors, including a poor-quality milk replacer and infectious causes like coccidiosis.

In summary, the first surge in the number of goats seroconverting with IgM antibodies to C. burnetii antigens was observed at 9 weeks of age, which underscores the need to vaccinate goats born on $C$. burnetii positive farms not later than 8 weeks of age. However, experimental studies are required to establish the effectiveness and feasibility of vaccinating goats at 8 weeks of age. It is expected that booster doses of vaccine will need to be administered to increase vaccine coverage. The shedding of $C$. burnettii by goats that remained seropositive after the initial seroconversion before the target breeding age provides more evidence supporting the notion that goats infected early in life can transmit $C$. burnetii to other goats and humans at their first kidding, underscoring the need to vaccinate young goats.

Post-weaning, the rate of seroconversion to C. burnetii was significantly higher within than outside the kidding seasons, this being more notable among goats in cohort 2, which were exposed to kidding season throughout the study period. Furthermore, goats from IgM seropositive does were two times more likely to seroconvert before the target breeding age, which points to either the occurrence of in utero transmission of C. burnetii or infection of goats during or shortly after birth.

Some of the goats that seroconverted before breeding shed C. burnetii at their first kidding, suggesting that goats infected early in life can be a risk for transmission of $C$. burnetii to susceptible animals in the herd. 
No statistically significant reductions in weight gain at the individual animal level were observed among goats that seroconverted against $C$. burnetii before breeding.

\section{Additional files}

Additional file 1. Kaplain-Meier survival curve showing the probability of intensively-reared kid goats remaining seronegative to C. burnetii before breeding, Victoria, Australia, 2015.

Additional file 2. Univariable assessment of the effect of risk factors on seroconversion against $C$. burnetii in goats.

Additional file 3. Multivariable Weibull accelerated failure time regression model assessing risk factors for seroconversion to C. burnetii before breeding in intensively-reared goats.

Additional file 4. Comparison of the rate of occurrence of antibody responses within and outside the kidding season in intensively managed goats.

Additional file 5. Median and inter-quartile range of antibody titres of goats in cohort 2 that shed C. burnetii at their first kidding during a $\mathrm{Q}$ fever outbreak farm.

\section{Competing interests}

The authors declare that they have no competing interests.

\section{Authors' contributions}

All authors participated in sample collection and analysis as well as in the writing of the manuscript. All authors read and approved the final manuscript.

\section{Acknowledgements}

We would like to acknowledge laboratory support from the Australian Rickettsial Reference Laboratory as well as support from staff at the affected farm and staff from the Mackinnon project.

\section{Funding}

No specific funding was provided for this work. All research was undertaken using resources at the institutions involved.

\section{Author details}

${ }^{1}$ Asia-Pacific Centre for Animal Health, Faculty of Veterinary and Agricultural Sciences, The University of Melbourne, Parkville, VIC 3010, Australia. ${ }^{2}$ The Mackinnon Project, Faculty of Veterinary and Agricultural Sciences, The University of Melbourne, Werribee, VIC 3010, Australia. ${ }^{3}$ Australian Rickettsial Reference Laboratory, Barwon Health, Geelong, VIC, Australia.

\section{Publisher's Note}

Springer Nature remains neutral with regard to jurisdictional claims in published maps and institutional affiliations.

Received: 8 March 2017 Accepted: 24 August 2017

Published online: 15 September 2017

\section{References}

1. Kampschreur LM, Hagenaars JC, Wielders CC, Elsman P, Lestrade PJ, Koning OH, Oosterheert JJ, Renders NH, Wever PC (2013) Screening for Coxiella burnetii seroprevalence in chronic $\mathrm{Q}$ fever high-risk groups reveals the magnitude of the Dutch $\mathrm{Q}$ fever outbreak. Epidemiol Infect 141:847-851

2. Schack M, Sachse S, Rödel J, Frangoulidis D, Pletz MW, Rohde GU, Straube E, Boden K (2014) Coxiella burnetii (Q fever) as a cause of communityacquired pneumonia during the warm season in Germany. Epidemiol Infect 142:1905-1910
3. Angelakis E, Edouard S, Lafranchi MA, Pham T, Lafforgue P, Raoult D (2014) Emergence of Q fever arthritis in France. J Clin Microbiol 52:1064-1067

4. Million M, Walter G, Thuny F, Habib G, Raoult D (2013) Evolution from acute $\mathrm{Q}$ fever to endocarditis is associated with underlying valvulopathy and age and can be prevented by prolonged antibiotic treatment. Clin Infect Dis 57:836-844

5. Levy PY, Carrieri P, Raoult D (1999) Coxiella burnetii pericarditis: report of 15 cases and review. Clin Infect Dis 29:393-397

6. Piquet P, Raoult D, Tranier P, Mercier C (1994) Coxiella burnetii infection of pseudoaneurysm of an aortic bypass graft with contiguous vertebral osteomyelitis. J Vasc Surg 19:165-168

7. Kampschreur LM, Delsing CE, Groenwold RHH, Wegdam-Blans MC, Bleeker-Rovers CP, de Jager-Leclercq MG, Hoepelman Al, van Kasteren ME, Buijs J, Renders NH et al (2014) Chronic Q fever in the Netherlands 5 years after the start of the $\mathrm{Q}$ fever epidemic: results from the Dutch chronic Q fever database. J Clin Microbiol 52:1637-1643

8. Briggs BJ, Raoult D, Hijazi ZM, Edouard S, Angelakis E, Logan LK (2016) Coxiella burnetii endocarditis in a child caused by a new genotype. Pediatr Infect Dis J 35:213-214

9. Francis JR, Robson J, Wong D, Walsh M, Astori I, Gill D, Nourse C (2016) Chronic recurrent multifocal Q fever osteomyelitis in children: an emerging clinical challenge. Pediatr Infect Dis J 35:972-976

10. Angelakis E, Mediannikov O, Socolovschi C, Mouffok N, Bassene H, Tall A, Niangaly H, Doumbo O, Znazen A, Sarih M (2014) Coxiella burnetii-positive PCR in febrile patients in rural and urban Africa. Int J Infect Dis 28:107-110

11. Chryssanthou E, Cuenca-Estrella M, Denning D (2006) Q fever in young children, Ghana. Mycopathologia 162:289-294

12. Delsing CE, Kullberg BJ (2008) Q fever in the Netherlands: a concise overview and implications of the largest ongoing outbreak. Neth J Med 66:365-367

13. Guigno D, Coupland B, Smith EG, Farrell ID, Desselberger U, Caul EO (1992) Primary humoral antibody response to Coxiella burnetii, the causative agent of Q fever. J Clin Microbiol 30:1958-1967

14. Smith DL, Ayres JG, Blair I, Burge PS, Carpenter MJ, Caul EO, Coupland B, Desselberger U, Evans M, Farrell ID et al (1993) A large Q fever outbreak in the West Midlands: clinical aspects. Respir Med 87:509-516

15. Lyytikäinen $\mathrm{O}$, Ziese T, Schwartländer B, Matzdorff P, Kuhnhen C, Jäger $C$, Petersen L (1998) An outbreak of sheep-associated Q fever in a rural community in Germany. Eur J Epidemiol 14:193-199

16. Hogerwerf $L$, van den Brom R, Roest HI, Bouma A, Vellema P, Pieterse $M$, Dercksen D, Nielen M (2011) Reduction of Coxiella burnetii prevalence by vaccination of goats and sheep, the Netherlands. Emerg Infect Dis 17:379-386

17. de Cremoux R, Rousset E, Touratier A, Audusseau G, Nicollet P, Ribaud D, David V, Le Pape M (2012) Coxiella burnetii vaginal shedding and antibody responses in dairy goat herds in a context of clinical $\mathrm{Q}$ fever outbreaks. FEMS Immunol Med Microbiol 64:120-122

18. Arricau-Bouvery N, Souriau A, Bodier C, Dufour P, Rousset E, Rodolakis A (2005) Effect of vaccination with phase I and phase II Coxiella burnetii vaccines in pregnant goats. Vaccine 23:4392-4402

19. de Cremoux R, Rousset E, Touratier A, Audusseau G, Nicollet P, Ribaud D, David V, Le Pape M (2012) Assessment of vaccination by a phase I Coxiella burnetii-inactivated vaccine in goat herds in clinical $\mathrm{Q}$ fever situation. FEMS Immunol Med Microbiol 64:104-106

20. Bontje DM, Backer JA, Hogerwerf L, Roest HI, van Roermund HJ (2016) Analysis of $\mathrm{Q}$ fever in Dutch dairy goat herds and assessment of control measures by means of a transmission model. Prev Vet Med 123:71-89

21. van Asseldonk M, Bontje DM, Backer JA, Roermund HJ, Bergevoet RH (2015) Economic aspects of Q fever control in dairy goats. Prev Vet Med 121:115-122

22. Rousset E, Durand B, Champion JL, Prigent M, Dufour P, Forfait C, Marois M, Gasnier T, Duquesne V, Thiéry R, Aubert MF (2009) Efficiency of a phase 1 vaccine for the reduction of vaginal Coxiella burnetii shedding in a clinically affected goat herd. Clin Microbiol Infect 15:188-189

23. Bond KA, Vincent G, Wilks CR, Franklin L, Sutton B, Stenos J, Cowan R, Lim K, Athan E, Harris O et al (2015) One health approach to controlling a Q fever outbreak on an Australian goat farm. Epidemiol Infect 144:1129-1141

24. Muleme M, Stenos J, Vincent G, Wilks CR, Devlin JM, Campbell A, Cameron A, Stevenson MA, Graves S, Firestone SM (2017) Peripartum dynamics of 
Coxiella burnetii infections in intensively managed dairy goats associated with a Q fever outbreak in Australia. Prev Vet Med 139(Pt A):58-66

25. Rousset E, Durand B, Berri M, Dufour P, Prigent M, Russo P, Delcroix T, Touratier A, Rodolakis A, Aubert M (2007) Comparative diagnostic potential of three serological tests for abortive $\mathrm{Q}$ fever in goat herds. Vet Microbiol 124:286-297

26. Roest HI, Post J, van Gelderen B, van Zijderveld FG, Rebel JM (2013) Q fever in pregnant goats: humoral and cellular immune responses. Vet Res 44:67

27. Thrusfield M (2013) Veterinary epidemiology. Elsevier, Amsterdam

28. Da Silva AS, Tonin AA, Camillo G, Weber A, Lopes LS, Cazarotto CJ, Balzan A, Bianchi AE, Stefani LM, Lopes STA, Vogel FF (2014) Ovine toxoplasmosis: indirect immunofluorescence for milk samples as a diagnostic tool. Small Rumin Res 120:181-184

29. Lockhart MG, Graves SR, Banazis MJ, Fenwick SG, Stenos J (2011) A comparison of methods for extracting DNA from Coxiella burnetii as measured by a duplex qPCR assay. Lett Appl Microbiol 52:514-520

30. Akaike H (1998) Information theory and an extension of the maximum likelihood principle. In: Selected papers of Hirotugu Akaike. Springer, Berlin. doi: 10.1007/978-1-4612-1694-0_15

31. Hubeaux S, Rufibach K (2014) SurvRegCensCov: Weibull regression for a right-censored endpoint with a censored covariate, arXiv preprint arXiv:1402.0432, https://arxiv.org/abs/1402.0432

32. R Core Team (2014) R: a language and environment for statistical computing. R Foundation for Statistical Computing. https://www.r-project.org/

33. Woldehiwet Z (2004) Q fever (coxiellosis): epidemiology and pathogenesis. Res Vet Sci 77:93-100

34. Berri M, Rousset E, Champion JL, Russo P, Rodolakis A (2007) Goats may experience reproductive failures and shed Coxiella burnetii at two successive parturitions after a Q fever infection. Res Vet Sci 83:47-52

35. Sánchez J, Souriau A, Buendía AJ, Arricau-Bouvery N, Martínez CM, Salinas J, Rodolakis A, Navarro JA (2006) Experimental Coxiella burnetii infection in pregnant goats: a histopathological and immunohistochemical study. J Comp Pathol 135:108-115

36. Roest HJ, van Gelderen B, Dinkla A, Frangoulidis D, van Zijderveld F, Rebel $J$, van Keulen $L$ (2012) Q fever in pregnant goats: pathogenesis and excretion of Coxiella burnetii. PLoS One 7:e48949

37. Palmer NC, Kierstead M, Key DW, Williams JC, Peacock MG, Vellend H (1983) Placentitis and abortion in goats and sheep in Ontario caused by Coxiella burnetii. Can Vet J 24:60-61

38. Bouvery NA, Souriau A, Lechopier P, Rodolakis A (2003) Experimental Coxiella burnetii infection in pregnant goats: excretion routes. Vet Res 34:423-433

39. Reichel R, Mearns R, Brunton L, Jones R, Horigan M, Vipond R, Vincent G, Evans S (2012) Description of a Coxiella burnetii abortion outbreak in a dairy goat herd, and associated serology, PCR and genotyping results. Res Vet Sci 93:1217-1224

40. Ben Amara A, Ghigo E, Le Priol Y, Lépolard C, Salcedo SP, Lemichez E, Bretelle F, Capo C, Mege JL (2010) Coxiella burnetii, the agent of Q fever, replicates within trophoblasts and induces a unique transcriptional response. PLoS One 5:e15315
41. Waag DM, Thompson HA (2005) Pathogenesis of and immunity to Coxiella burnetii. In: Biological weapons defense, Springer, pp 185-207.

42. Moore JD, Barr BC, Daft BM, O'Connor MT (1991) Pathology and diagnosis of Coxiella burnetii infection in a goat herd. Vet Pathol 28:81-84

43. Rodolakis A (2014) Zoonoses in goats: how to control them. Small Rumin Res 121:12-20

44. Rodolakis A, Berri M, Héchard C, Caudron C, Souriau A, Bodier CC, Blanchard B, Camuset P, Devillechaise P, Natorp JC et al (2007) Comparison of Coxiella burnetii shedding in milk of dairy bovine, caprine, and ovine herds. J Dairy Sci 90:5352-5360

45. Moreno-Indias I, Sánchez-Macías D, Castro N, Morales-delaNuez A, Hernández-Castellano LE, Capote J, Argüello A (2012) Chemical composition and immune status of dairy goat colostrum fractions during the first 10h after partum. Small Rumin Res 103:220-224

46. Peacock MG, Fiset P, Ormsbee R, Wisseman CL Jr (1979) Antibody response in man following a small intradermal inoculation with Coxiella burnetii phase I vaccine. Acta Virol 23:73-81

47. Powell OW, Stallman ND (1962) The incidence and significance of phase 1 complement-fixing antibody in Q fever. J Hyg 60:359-364

48. Krumbiegel ER, Wisniewski HJ (1970) Q fever in the Milwaukee area. II. Consumption of infected raw milk by human volunteers. Arch Environ Health 21:63-65

49. European Food Safety Association (2010) Q fever. EFSA J 8:1595-1709

50. Kurmann J, Sydler T, Brugnera E, Buergi E, Haessig M, Suter M, Sidler X (2011) Vaccination of dams increases antibody titer and improves growth parameters in finisher pigs subclinically infected with porcine circovirus type 2. Clin Vaccine Immunol 18:1644-1649

51. Niewiesk S (2014) Maternal antibodies: clinical significance, mechanism of interference with immune responses, and possible vaccination strategies. Front Immunol 5:446

52. Pomorska-Mól M, Markowska-Daniel I (2010) Interferon- $\gamma$ secretion and proliferative responses of peripheral blood mononuclear cells after vaccination of pigs against Aujeszky's disease in the presence of maternal immunity. FEMS Immunol Med Microbiol 58:405-411

53. Toman R, Heinzen RA, Samuel JE, Mege JL (2012) Components of protective immunity, Coxiella burnetii: recent advances and new perspectives in research of the Q fever bacterium. Springer, Berlin, pp 91-104

54. Corpa JM, Pérez V, García Marín JF (2000) Differences in the immune responses in lambs and kids vaccinated against paratuberculosis, according to the age of vaccination. Vet Microbiol 77:475-485

55. Alsaleh A, Fieni F, Rodolakis A, Bruyas JF, Roux C, Larrat M, Chatagnon G, Pellerin JL (2013) Can Coxiella burnetii be transmitted by embryo transfer in goats? Theriogenology 80:571-575

56. Maurin M, Raoult D (1999) O fever. Clin Microbiol Rev 12:518-553

57. Angelakis E, Raoult D (2010) Q fever. Vet Microbiol 140:297-309

58. Tigertt WD, Benenson AS, Gochenour WS (1961) Airborne Q fever. Bacteriol $\operatorname{Rev}$ 25:285-293

\section{Submit your next manuscript to BioMed Central and we will help you at every step:}

- We accept pre-submission inquiries

- Our selector tool helps you to find the most relevant journal

- We provide round the clock customer support

- Convenient online submission

- Thorough peer review

- Inclusion in PubMed and all major indexing services

- Maximum visibility for your research

Submit your manuscript at www.biomedcentral.com/submit
BioMed Central 